\title{
Pseudozyma hubeiensis, an unexplored yeast: It's potential in biomass conversion to value added products
}

\begin{abstract}
The species of the genus Pseudozyma belong to ustilaginales based on the studies on morphology and molecular characterization. There are 11 species reported so far from this genus that differ from one another in the sequences of ITS and D1/D2 regions. Eleven species of Pseudozyma reported so far were studied for assimilation reactions which differentiated all the species of the genus Pseudozyma from one another. Almost all the species of the Pseudozyma are able to assimilate inositol but $P$. hubeiensis shows negative inositol assimilation reaction. There are no reports available for the production of industrially important enzymes and other biological products from $P$. hubeiensis and hence it remained still unexplored. $P$. hubeiensis was first isolated from sandal wood in our laboratory which produced a complete cellulase free xylanolytic system consisting of endoxylanase and $\beta$-xylosidase. The purified xylanases produced smaller chain length (X3$\mathrm{X} 7)$ xylooligosaccharides (XOS) which have great potential use as prebiotic in functional food. In addition, it is also used in cosmetics, pharmaceuticals or agricultural products and as a plant growth regulator. It also produces high amounts of metal and ethanol tolerant $\beta$-xylosidase qualifying its use in lignocellulosic biomass hydrolysis when mixed with enzyme preparations that are deficient in $\beta$-xylosidase. $P$. hubeiensis utilizes simultaneously all the sugars present in biomass hydrolysate and convert them to lipids. In this mini-review, the biology and enzymology underlying the biomass degrading enzymes and the production of lipids/biosurfactants especially MELs are described. An approach to developing $P$. hubeiensis strains for production of biomass degrading enzymes and their application is outlined.
\end{abstract}

Keywords: pseudozyma hubeiensis, Endoxylanase, $\beta$-xylosidase, Xylooligosaccharides (XOS), Biomass degradation, mannosylerythritol lipids (MELs)
Volume 6 Issue 2 - 2018

\author{
Nutan Mhetras, Digambar Gokhale \\ NCIM Resource Center, CSIR-National Chemical Laboratory, \\ India
}

Correspondence: Digambar Gokhale, NCIM Resource Center, CSIR-National Chemical laboratory, Pune 4 | I008, India, Tel +9| 202590 2452, Fax 91-20-2590267l, Email dv.gokhale@ncl.res.in, dr.nutan.c.mhetras@gmail.com

Received: December 26, 2017 | Published: March 09, 2018

\section{The genus pseudozyma}

The species of the genus Pseudozyma belong to ustilaginales based on the morphological studies ${ }^{1}$ and molecular characterization. ${ }^{2,3}$ There are 11 species reported so far from this genus that are distinguished by analysing the sequences of combined ITS and D1/D2 regions. Boekhout \& Fell ${ }^{4}$ reported seven species and Sugita et al. ${ }^{5}$ described two species isolated from blood of the patients. Two more species of Pseudozyma were isolated from wilting leaves of different plants that are named as Pseudozyma hubeiensis and Psedozyma shanxiensis. ${ }^{6}$ These species are distinguished from other reported species by morphological studies and physiological characterization. The novelty of these two species was also confirmed by molecular taxonomic analysis based on sequencing of 26S rRNA gene, D1/ D2 domain and internal transcribed spacer (ITS) regions (6). All 11 species of Pseudozyma were further studied for assimilation reactions which differentiated all the species of the genus Pseudozyma from one another. The ability to grow at $40^{\circ} \mathrm{C}$ and no assimilation of erythritol are the characteristics that differentiate $P$. shanxiensis from all other species of Pseudozyma. However, P. hubeiensis does not assimilate inositol and this characteristic differentiates $P$. hubeiensis from all other species. ${ }^{6} P$. hubeiensis was first isolated from decaying sandal wood in our laboratory ${ }^{7}$ and then it was sent for identification to National Collection of Yeast Cultures (NCYC) in 2008. The sequencing of 26 rDNA D1/D2 domain and standard taxonomic tests confirmed that it is a novel strain of P. hubeiensis. It was then deposited in NCIM Resource Center, CSIR-National Chemical Laboratory, Pune, with an accession number NCIM 3574.

Among all species of Pseudozyma, Pseudzyma antarctica (Formally known as Candida antarctica) has been a most studied. It is well known producer of glycolipid, manosylerythritol (MEL) from vegetable oil including soybean oil, alkanes, glycerol, glucose and xylose $^{8}$ and also from cellulosic materials. ${ }^{9}$ MELs show excellent surface active properties in addition to versatile biochemical actions. In addition to its bio-surfactant property, MEL possesses antitumor and cell differentiation induction activities. ${ }^{10}$ The other species such as P. aphidis, P. rugulosa, P. fusiformata are also known to produce MEL. ${ }^{11}$ Extracellular esterases ${ }^{12}$ and biodegradable plastic degrading enzymes ${ }^{13}$ have been reported from $P$. antarctica. The plastic degrading enzyme named $\mathrm{PaE}$ produced by $P$. antarctica degrades biodegradable plastic films composed of poly (butylele succinate) (PBS), poly (butylene succinate-co-adipate) (PBSA) and poly (lactic acid) (PLA). Xylose induced xylanases were reported from $P$. antarctica and the $33 \mathrm{kDa}$ purified protein was found to produce xylose from xylan indicating that they are endo-xylanases. ${ }^{14}$ Genome and transcriptome analysis of $P$. antarctica was recently reported. ${ }^{15}$ The another novel yeast species, $P$. brasiliensis produced the xylan induced secretome containing endo-xylanase and $\beta$-xylosidase. ${ }^{16}$ 


\section{Enzymes and lipids produced by Pseudozyma hubeiensis}

Though Basidiomycetes yeasts are essential for carbon cycle, the production of biomass degrading enzymes by basidiomycetes yeasts still remained unexplored compared to Ascomycetes. Wide range of enzymes produced by a microbial strain displays its ability to adapt to natural habitats and hence new species may emerge through such adaptations. Such adapted microorganisms have the ability to produce altogether new enzymes of industrial importance. The Basidiomycetes yeasts belonging to the genus Pseudozyma have immense potential in industrial processes but they are not still properly explored. The knowledge of the ability of Pseudozyma species to degrade biomass polysaccharides is very limited. P. hubeiensis was first isolated from sandal wood in our laboratory in 1992 which was found to produce a complete xylanolytic system consisting of cellulase free xylanase and $\beta$-xylosidase. ${ }^{17}$ The crude xylanase produced by $P$. hubeiensis degraded xylan from different agricultural waste materials to produce xylose which can be the starting material for production of value added chemicals. ${ }^{18}$ Such cellulase free xylanases can be used in paper and pulp industries to remove xylan in paper and pulp in place of chlorine which is being used in bio-bleaching process.

Xylo-oligosaccharides (XOS) acting as prebiotics are the most preferred dietary fibres or functional foods. The commercial importance of non-digestible XOS is based on their beneficial properties. XOS stimulate the growth and activity of limited number of bacteria such as Bifidobacterium and Lactobacillus present in the colon and also suppress the activity of entero-putretive and pathogenic bacteria. In addition, they are moderately sweet with organoleptic characteristics suitable for use as supplements in foods. In addition, they show stability at wide $\mathrm{pH}$ range and temperature which make them most suitable dietary supplementation. ${ }^{19-21}$ Apart from prebiotic and bulking agents, XOS are used as stabilisers in cosmetics, immunestimulating agents, and antioxidant in pharmaceuticals. XOS is also known to decrease the blood lipids, protect the liver functions and decrease the blood pressure. It was found that the supplementation of XOS was successful in inhibiting the precancerous lesions and also in lowering the colonel $\mathrm{pH}$ value..$^{22,23}$ Therefore, diets supplemented with XOS have beneficial effect in improving gastrointestinal health. Furthermore, XOS were found to be more efficient than other prebiotics such as fructo-oligosaccharides in dietary supplementation. The preventive effect of XOS against contact hypersensitivity was also investigated in mice. ${ }^{24} P$. hubeiensis NCIM 3574 produces two distinct xylanases which were purified to homogeneity by DEAE cellulose chromatography followed by Sephadex G-50 column chromatography. Molecular masses of two native xylanases were $33.3 \mathrm{kDa}(\mathrm{PhX} 33)$ and $20.1 \mathrm{kDa}(\mathrm{PhX} 20)$ which are confirmed by MALDI-TOF mass spectrometry and also SDS-PAGE. The CD spectra analysis revealed that $\mathrm{PhX} 33$ has predominantly $\alpha$-helix and $\mathrm{PhX} 20$ contained predominantly $\beta$-sheets. The chemical modification studies revealed that the $\mathrm{PhX} 33$ has the active site consisting of three tryptophan and one carboxyl residues. The active site of PhX20 is comprised of tryptophan, carboxyl and histidine with one residue each. Carboxyl residue is mainly involved in catalysis and tryptophane residues are solely involved in substrate binding. Histidine residue present at the active site of $\mathrm{PhX} 20$ appeared to have a role in substrate binding. LC-MS/MS ion search of tryptic digestion of these xylanases revealed that there is no significant homology of peptides with known fungal xylanase sequences which indicate that these xylanases appear to be new $\mathrm{PhX} 33$ hydrolyzed xylan into xylotriose, xylotetraose and xylopentaose and PhX20 hydrolyzed xylan into xylotriose, xylotetraose, xylopentaose, xylohexaose and xyloheptaose. No xylose and xylobiose were detected in the hydrolyzates. Both the xylanases produced only xylooligosaccharides (XOS) with degree of polymerization (DP) 3 to $7 .{ }^{25}$ The complete utilization of biomass to obtain bulk chemicals and XOS makes these enzymes very interesting from industrial perspective.

The $\beta$-xylosidase from an unexplored yeast, Pseudozyma hubeinsis was induced by birchwood xylan when the yeast was grown at $27^{\circ} \mathrm{C}$. The enzyme was purified to homogeneity and it was found to be a glycoprotein with $23 \%$ glycosylation. The purification protocol involved ammonium sulphate precipitation, QAE-sephadex A50 ion exchange chromatography and sephacryl-200 column chromatography which resulted in 8.3 fold purification with $53.12 \%$ final recovery. It is a monomeric protein of $110 \mathrm{kDa}$ molecular weight confirmed by both SDS-PAGE and MALDI-TOF mass spectrometry. The enzyme was active at $60^{\circ} \mathrm{C}$ and $\mathrm{pH} 4.5$ and stable at $\mathrm{pH}$ range (4-9) and at $50^{\circ} \mathrm{C}$ for $4 \mathrm{~h}$. Its active site is comprised of carboxyl, tyrosine and tryptophan residues. The carboxyl residue is involved in catalysis and tryptophan residue is solely involved in substrate binding. The best match from the search of the NCBInr database was with gi| 808364558 glycoside hydrolase of Pseudozyma hubeiensis SY62 with 26\% sequence coverage confirming that it is a glycoside hydrolase/beta-glucosidase. From the search of customized SWISSPROT database, it was revealed that SWISSPROT does not contain any entries that are similar to the purified enzyme. The properties such as high catalytic performance, significant stability and activity at acidic $\mathrm{pH}$ and high temperatures, high metal and ethanol tolerance qualify this enzyme for use in the hydrolysis of lignocellulosic biomass when mixed with efficient and suitable enzyme complexes since most of the commercial enzyme preparations are deficient in $\beta$-xylosidases. ${ }^{17}$

Lipids accumulated by oleaginous yeasts is viewed as promising source for second generation biodiesel since the fatty acid compositions produced by these yeast is suitable for biodiesel production. These yeasts grow faster compared to other oleaginous microorganisms and have the potential to convert various carbon sources such as glucose, xylose, starch, Cellobiose to lipids. ${ }^{26-28}$ Exhaustive screening of large number of yeast isolates led to the discovery of Pseudozyma hubeiensis IPM1-10 that converts sugar mixtures consisting of glucose, xylose and arabinose to lipids. ${ }^{29}$ Oleaginous microbes utilise xylose exclusively by phosphoketolase pathway resulting in lower yields of lipids. ${ }^{30}$ Due to the absence of glucose repression in $P$. hubeiensis, this yeast will turn out to be a potential candidate for second generation biodiesel production from hydrolysate of cellulosic biomass. The mechanism of absence of glucose repression still remained unknown and further study is essential to unravel the sugar assimilation mechanism in $P$. hubeiensis. Sari et al. ${ }^{31}$ isolated $P$. hubeiensis Y10BS025 that produced high levels $(115 \mathrm{~g} / \mathrm{L})$ of mannosylerythritol lipids (MELs) during growth in a medium containing yeast extract and soybean oil after 8 days of fermentation with improved shaking conditions. P. hubeiensis produces lipase by assimilating oils and secretes bio-surfactants. ${ }^{32,33}$ P. hubeiensis also can convert sugars such as glucose, xylose and arabinose to lipids and hence it has a great potential for utilization of 
unused biomass sugars and low cost raw materials. The potential of this novel yeast strain for production of value added products from renewable biomass is given in Figure 1.

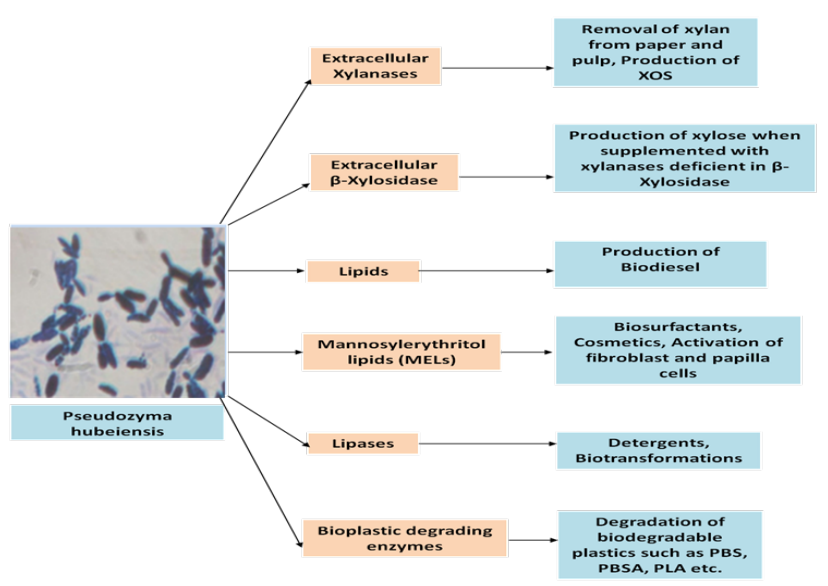

Figure I The potential of Pseudozyma hubeiensis for production of value added products from renewable biomass.

\section{Conclusion and future perspective}

P. hubeiensis produces a complete cellulase free xylanolytic enzyme complex consisting of both xylanases and $\beta$-xylosidase. Such cellulase free xylanases have been efficiently used in paper and pulp industries in place of chlorine to remove xylan for improving biobleaching processes. The purified xylanases produced smaller chain length (X3-X7) XOS which have great potential use as ingredients of functional food, cosmetics, pharmaceuticals or agricultural products and as a plant growth regulator. It also produces high amounts of metal and ethanol tolerant $\beta$-xylosidase qualifying its use in lignocellulosic biomass hydrolysis when mixed with enzyme preparations that are deficient in $\beta$-xylosidase. P. hubeiensis utilizes simultaneously all the sugars present in biomass hydrolysate and convert them to lipids thus posing to be a potential candidate for second generation biodiesel production. Competitive production of chemicals and fuels from biomass requires generation of revenue from each component (cellulose, hemicellulose and lignin) of lignocellulosic biomass. Cellulose, hemicellulose and lignin have different structures and chemistries and hence the effective fractionation to separate individual components in to their native state is very critical. Alonso et al ${ }^{34}$ used $\gamma$-valerolactone (GVL)/water/acid mixture to dissolve hemicellulose and lignin leaving behind high purity cellulose. Under the condition, lignin can be separated with high purity and hemicellulose can be converted to furfural. This hemicellulose fraction can be used for the production of xylanases to be used for XOS production. In addition, the hemicellulose fraction can be used to grow P. hubeiensis to get microbial biomass with high lipid content which can be diverted for second generation biodiesel production. It is essential to look for other enzymes such as lipases, esterases which may prove to be novel and efficient in performing chemically difficult transformations of organic compounds to drugs and drugs intermediates. Work is in progress in our laboratory in search of such enzymes in P. hubeiensis and assessment of their ability to perform unconventional bio-transformations and to degrade biodegradable plastics such as PBS, PBSA or PLA.

\section{Acknowledgements}

The authors gratefully acknowledge the support Emeritus Scientist scheme from Council of Scientific \& Industrial Research (CSIR), New Delhi, India.

\section{Conflict of interest}

There is no conflict to publish our article in this Journal.

\section{References}

1. Boekhout T. Systematics of anamorphs of Ustilaginales (smut fungi) - a preliminary survey. Stud Mycol. 1987;30:137-149.

2. Boekhout T. Pseudozyma bandoni emend. Boekhout, a genus for yeastlike anamorphs of ustilaginales. J Gen App Microbiol. 1995;41:359-366.

3. Fell JW, Boekhout T, Fonseka A, et al. Biodiversity and systematics of basidiomycetous yeasts as determined by large subunit rDNA D1/ D2domain sequence analysis, Int J Syst Evol Microbiol. 2000;50(Pt 3):1351-1371.

4. Boekhout T, Fell JW. Pseudozyma bandoni emend. Boekhout and a comparison with the yeast state of Ustilago maydis (De Candolle) Corda. In: Kurtzman CP, Fell JW, editors. The Yeast, a taxonomic study. 4th ed. Amsterdam: Elsevier; 1998: 790-797.

5. Sugita T, Takashima M, Poonwan N, et al. The first isolation ustilaginomycetous anamorphic yeasts, Pseudozyma species, from patients' blood and a description of two new species: $P$. parantarctica and P. thailandica. Microbiol Immunol. 2003;47(3):183-190.

6. Wang QM, Jia JH, Bai FY. Pseudominas hubeiensis sp. nov. and Pseudomonas shanxiensis sp. nov., novel ustilaginomycetous anamorphic yeast species from plant leaves, Int J Syst Evol Microbiol. 2006;56(Pt 1):289-293.

7. Bastawde KB, Puntambekar US, Gokhale DV. Optimization of cellulase free xylanase production by a novel yeast strain. $J$ Ind Microbiol. 1994;13(4):220-224.

8. Morita T, Fukuoka T, Imura T, et al. Production of mannosylerythritol lipids and their application in cosmetics. Appl Microbiol Biotechnol. 2013;97(11):4691-4700.

9. Faria NT, Santos M, Ferreira C, et al. Conversion of cellulosic materials into glycolipid biosurfactants, mannosylerythritol lipids, by Pseudozyma spp. Under SHFand SSF processes. Microb Cell Factories. 2014;13:55.

10. Isoda H, Shinmoto H, Kitamoto D, et al. Differentiation of human promyelocytic leukemia cell line HL60 by microbial extracellular glycolipids. Lipids. 1997;32(3):263-271.

11. Paulino BN, Pessoa MG, Mano MCR, et al. Current status in biotechnological production and applications of glycolipid biosurfactants. Appl Microbiol Biotechnol. 2016;100(24):10265-10293.

12. Ueda H, Mitsuhara I, Tabata J, et al. Extracellular esterases of phylloplane yeast Pseudozyma antarctica induce defect on cuticle layer structure and water-holding ability of plant leaves. Appl Microbiol Biotechnol. 2015;99(15):6405-6415.

13. Shinozaki Y, Morita T, Cao X, et al. Biodegradable plastic-degrading enzyme from Pseudozyma antarctica: cloning, sequencing, and characterization. Appl Microbiol Biotechnol. 2013;97(7):2951-2959.

14. Watanabe T, Suzuki K, Sato I, et al. Simultaneous bioethanol distillery wastewater treatment and xylanase production by the phyllosphere yeast Pseudozyma antarctica GB-4(0). AMB Express. 2015;5:36. 
15. Morita $\mathrm{T}$, Koide $\mathrm{H}$, Hagiwara $\mathrm{H}$, et al. Genome and transcriptone analysis of the basidiomycetous yeast Pseudozyma hubeiensis producing extracellular glycolipid, mannosylerythritol lipids. PLoS One. 2014;9(2):e86490.

16. Neto AAK, Borin GP, Goldman GH, et al. Insights into the plant polysaccharide degradation potential of the xylanolytic yeast Pseudozyma brasiliensis. FEMS Yeast Res. 2016;16(2):fov117.

17. Mhetras N, Liddel S, Gokhale D. Purification and characterization of an extracellular $\beta$-xylosidase from Pseudozyma hubeiensis NCIM 3574 (PhXyl), an unexplored yeast. AMB Express. 2016;6(1):73.

18. Gokhale DV, Patil SG, Bastawde KB. Potential application of yeast cellulase-free xylanase in agrowaste materials to remove hemicellulose fractions. Bioresource Technol. 1998;63(2):187-191.

19. Gibson GR, Roberfroid MB. Dietary modulation of the human colonic microbiota: introducing the concept of prebiotics. J Nutrition. 1995;125(6):1401-1412.

20. Katapodis P, Kavarnou A, Kintzios S, et al. Production of acidic xylooligosaccharides by a family 10 endoxylanase from Thermoascus aurantiacus and use as plant growth regulators. Biotechnol Lett. 2002;24(17):1413-1416.

21. Barreteau H, Delattre C, Michaud P. Production of oligosaccharides as promising new food additive generation. Food Technol Biotechnol. 2006; $44: 323-333$

22. Hsu CK, Liao JW, Chung YC, et al. Xylooligosaccharides and fructooligosaccharides affect the intestinal microbiota and precancerous colonic lesion Development in rats. J Nutrition. 2004;134(6):1523-1528.

23. Moure A, Gullon P, Dominguez H, et al. Advances in the manufacture, purification and applications of xylo-oligosaccharides as food additives and nutraceuticals. Proc Biochem. 2006;41(9):1913-1923.

24. Yoshino K, Higashi N, Koga K. Preventive effect of acidic xylooligosaccharide on contact hypersensitivity in mice. $J$ Health Science. 2006;52:628-632.
25. Adsul MG, Bastawde KB, Gokhale DV. Biochemical characterization of two xylanases from yeast Pseudozyma hubeiensis producing only xylooligosaccharides. Bioresour Technol. 2009;100(24):6488-6495.

26. Gong Z, Wang Q, Shen H, et al. Co-fermentation of Cellobiose and xylose by Lipomyces starkeyi for lipid production. Bioresour Technol. 2012;117:20-24.

27. Huang C, Xf Chen, Xiong L, et al. Single cell oil production from lowcost substrates: the possibility and potential of its industrialization. Biotechnol Adv. 2013;31(2):129-39.

28. Tanimura A, Takashima M, Sugita T, et al. Cryptococcus terricola is a promising oleaginous yeast for biodiesel production from starch through consolidated bioprocessing. Sci Rep. 2014;4:4776.

29. Tanimura A, Takashima M, Sugita T, et al. Lipid production through simultaneous utilization of glucose, xylose, and L-arabinose by Pseudozyma hubeiensis: a comparative screening study. AMB Express. 2016;6(1):58.

30. Papanikolaou S, Aggelis G. Lipids of oleaginous yeasts. Part I: biochemistry of single cell oil production. Eur J Lipid Sci Technol. 2011;113(8):1031-1051.

31. Sari M, Kanti T, Made Artika I, et al. Identification of Pseudozyma hubeiensis Y10BS025 as a potent producer of glycolipid bio-surfactant mannosylerythritol lipids. Am J Biochem Biotechnol. 2013;9(4):430 437.

32. Bussamara R, Fuentefria AM, de Oliveira ES, et al. Isolation of a lipase-secreting yeast for enzyme production in a pilot-plant scale batch fermentation. Bioresource Technol. 2010;101(1):268-275.

33. Bussamara R, Dall'agnol L, Schrank A, et al. Optimal conditions for continuous immobilization of Pseudozyma hubeiensis (strain HB85A) lipase by adsorption in packed-bed reactor by response surface methodology. Enzyme Res. 2012;2012:320178.

34. Alonso DM, Hakim SK, Zhou S, et al. Increasing the revenue from lignocellulosic biomass: Maximizing feedstock utilization. Sci Adv 2017;3(5):e1603301. 